\title{
Remarks on the Lattice Green Function for the Anisotropic Face Centered Cubic Lattice
}

\author{
J.H. Asad ${ }^{a} *$, R.S. Hijuawi ${ }^{b}$, Eyad Hasan Hasan ${ }^{c}$, A.A. Diab ${ }^{d}$ And J.M. Khalifeh ${ }^{e}$ \\ ${ }^{a}$ Dep. of Physics, Faculty of Sciences, Palestine Technical University, Tulkarm, P.O. Box 7, Palestine \\ ${ }^{b}$ Dep. of Physics, College of Sciences, Mutah University, Karak, Jordan \\ ${ }^{c}$ Applied Physics Department, Faculty of Science, Tafila Technical University, P.O. Box 179, Tafila 66110, Jordan \\ ${ }^{d}$ General Studies Dep., Yanbu Industrial College, P.O. Box 30436, Yanbu Industrial City, KSA \\ ${ }^{e}$ Dep. of Physics, Jordan University, Amman 11942, Jordan \\ (Received July 19, 2015; in final form October 15, 2015)
}

An expression for the Green function of anisotropic face centered cubic lattice is evaluated analytically and numerically for a single impurity problem. The density of states, phase shift and scattering cross-section are expressed in terms of complete elliptic integrals of the first kind.

DOI: 10.12693/APhysPolA.129.52

PACS: 61.50.Ah, 05.50.+q, 04.60.Nc, 63.90.+t, 02.90. $+\mathrm{p}$

\section{Introduction}

The lattice Green function (LGF) is defined as [1]:

$$
G(E)=\frac{\Omega}{(2 \pi)^{d}} \int_{1 \mathrm{BZ}} \frac{F(\boldsymbol{k})}{E-E(\boldsymbol{k})} \mathrm{d} \boldsymbol{k}
$$

where $E(\boldsymbol{k})$ is a dispersion relation, $F(\boldsymbol{k})$ is an appropriate function with a general expression $F(\boldsymbol{k})=\mathrm{e}^{\mathrm{i} \boldsymbol{k}\left(\boldsymbol{r}-\boldsymbol{r}^{\prime}\right)}$ where $r$ and $r^{\prime}$ represent lattice sites, and it is a solution of the homogeneous Helmholtz equation, $\Omega$ is the volume of the crystal in real space, $d$ is the dimension, and $1 \mathrm{BZ}$ denotes that the integration is restricted to the first Brillouin zone $[1,2]$.

The LGF is a basic function in the study of the solid state physics and condensed matter [3]. Green was the first physicist who established the basic concepts of Green's function (GF) in the potential theory, and his work was focused on solving Laplace's and Poisson's equations with different boundary conditions. The use of GF method plays an important role in many-body problems [4], especially in problems of solid state physics where an enormous progress has been realized. In the mathematical problem of quantum theory which consists of solving linear operator equations with given boundary conditions, GF constitutes the natural language to study boundary conditions.

Nowadays, GF is one of the most important concepts in many branches of physics, as many quantities in solid state physics can be expressed in terms of the LGF. In the following there are some examples: statistical model of ferromagnetism such as the Ising model [5], the Heisenberg model [6], spherical model [7], random walk theory $[8,9]$, diffusion [10], band structure [11], and in recent

\footnotetext{
*corresponding author; e-mail: drjasad@yahoo.com
}

years it becomes an important tool in analyzing infinite electric networks [12-26].

The LGF for several structure lattices has been widely studied during the second half of the last century. The LGF for the rectangular lattice has been investigated by Katsura and Inawashiro [27]; they used the MellinBarnes type integral. Recurrence relation, which gives the LGF along the diagonal direction from a couple of values of complete elliptic integrals of the first and second kinds for the rectangular and square lattices, has been derived by Morita [28].

The LGF for simple cubic (sc) lattice at the origin $G(0,0,0)$ has been investigated by many authors: Joyce [29] expressed $G(0,0,0)$ in terms of the complete elliptic integrals of the first kind, Horiguchi [30] expressed $G(1,0,0)$ as a sum of simple integrals of the complete elliptic integrals of the first kind and evaluated it numerically, Katsura et al. [31, 32] investigated the LGF for many lattices, and finally, Glasser and Boersma [33] showed that $G(l, m, n)$ can be expressed rationally in terms of $G(0,0,0)$.

The LGF for the face centered cubic (fcc) lattice was studied well by Iwata [34], he expressed $G(0,0,0)$ in a compact form as a product of complete elliptic integrals of the first kind. The LGF at any lattice site $G(l, m, n)$ was studied by Mano [35]; $G(l, m, n)$ is expressed in terms of linear combinations of complete elliptic integrals of the first and second kind. In their paper Glasser and Boersma [33] expressed the LGF for fcc lattice rationally in terms of the known value of $G(0,0,0)$.

Finally, Hijjawi et al. [36-39] evaluated analytically and numerically GF, density of states, phase shift, and scattering cross-section for different lattices.

In this paper we report on the single impurity LGF.

The paper is organized as follows: Sect. 2 is devoted to the general definition of the diagonal LGF and its form inside and outside the band for anisotropic fcc lattice in terms of the complete elliptic integrals of the first kind. 
Elliptic integrals were studied well by many authors [40]. This section also contains the formulae for the density of states, phase shift and scattering cross-section for a point defect case. In Sect. 3 we present the results and discussion.

\section{The anisotropic fcc lattice Green function}

In this section we are going to study the anisotropic fcc lattice. As it is known if we measure any physical quantities (such as conductivity) and always obtaining the same result irrespective of the direction then we have isotropic sample, but if the measured physical quantities differ with orientation then we are talking about anisotropic sample.

The GF is usually represented by a matrix operator with diagonal elements and non-diagonal. Each site of the lattice represents a state, so the elements of the matrix takes the form $G\left(\boldsymbol{r}, \boldsymbol{r}^{\prime}\right)$ where $\boldsymbol{r}$ and $\boldsymbol{r}^{\prime}$ are two different lattice sites.

The diagonal GF for the anisotropic fcc lattice with nearest neighbor interaction is defined as [41-47]:

$$
\begin{aligned}
& G^{0}(E)=G^{0}(L, L ; E)=\frac{1}{\pi^{3}} \int_{0}^{\pi} \int_{0}^{\pi} \int_{0}^{\pi} \mathrm{d} k_{x} \mathrm{~d} k_{y} \mathrm{~d} k_{z} \\
& \quad \times\left(E-a_{1} \cos \left(k_{x}\right) \cos \left(k_{y}\right)-a_{2} \cos \left(k_{x}\right) \cos \left(k_{z}\right)\right. \\
& \left.\quad-a_{3} \cos \left(k_{y}\right) \cos \left(k_{z}\right)\right)^{-1}
\end{aligned}
$$

where $E>2 a_{1}+a_{3}$.

We shall consider the interactions between the nearest neighbors $a_{1}$ in $x y, a_{2}$ in $x z$ cubic planes and the interactions between the nearest neighbors $a_{3}$ in $y z$ planes.

For the anisotropic fcc lattice diagonal GF means that $\boldsymbol{r}=\boldsymbol{r}^{\prime}$, so in this case $F(\boldsymbol{k})=1$, and we may write $G^{0}(L, L ; E)=G^{0}(E)$, so this is due to that the right hand side of the above equation is independent of $L$.

Integrating the above equation and using the method of analytic continuation [44-46], the diagonal GF outside the band is real and has the form (for $a_{1}=a_{2}$ ):

$$
G^{0}(E)=\frac{4}{\pi^{2}\left(E+a_{3}\right)} K\left(k_{+}\right) K\left(k_{-}\right),
$$

where $E>2 a_{1}+a_{3}$, and $k_{ \pm}^{2}=\frac{1}{2}\left[\left(1 \mp 4 \sqrt{\frac{a_{3}}{a_{1}^{2}} E\left(1+\frac{a_{3}}{a_{1}^{2}} E\right)}\right.\right.$

$/\left(E+a_{3}\right)^{2}-\sqrt{\left(E+a_{3}\right)^{2}-4 \frac{a_{3}}{a_{1}^{2}} E} \sqrt{\left(E+a_{3}\right)^{2}-4\left(1+\frac{a_{3}}{a_{1}^{2}} E\right)}$ $\left./\left(E+a_{3}\right)^{2}\right]$.

The GF outside the band is real, and we have a bound state, while inside the band GF is imaginary and they can be written as (see Appendix A):

or

$$
G^{0}(E)=\frac{4}{\pi^{2}\left(E+a_{3}\right)} K\left(k_{+}\right) K\left(k_{-}\right), E>2 a_{1}+a_{3},
$$

$$
\begin{aligned}
& G^{0}(E)=\frac{2}{\pi^{2}\left(E+a_{3}\right)}\left[\left(X_{+}^{2}+1\right)\left(X_{-}^{2}+1\right)\right]^{\frac{-1}{4}} \\
& \quad \times\left(K\left(v_{+}\right) K\left(u_{-}\right)+K\left(v_{-}\right) K\left(u_{+}\right)+i\left[K\left(v_{+}\right) K\left(u_{+}\right)\right.\right. \\
& \left.\left.\quad-K\left(v_{-}\right) K\left(u_{-}\right)\right]\right), \quad-\left(2 a_{1}-a_{3}\right)<E<0,
\end{aligned}
$$

$$
\begin{gathered}
X_{\mp}=\frac{\sqrt{-E}}{\left(E+a_{3}\right)^{2}} \\
\times\left(-\sqrt{\frac{\left[\left(E+a_{3}\right)^{2}-4 \frac{a_{3}}{a_{1}^{2}} E\right]\left[4\left(1+\frac{a_{3}}{a_{1}^{2}} E\right)-\left(E+a_{3}\right)^{2}\right]}{-E}}\right. \\
\mp 4 \sqrt{\left.\frac{a_{3}}{a_{1}^{2}}\left(1+\frac{a_{3}}{a_{1}^{2}} E\right)\right)} \\
\text { and } \\
v_{ \pm}^{2}=\frac{1}{2}\left(1 \pm \sqrt{\frac{X_{-}^{2}}{X_{-}^{2}+1}}\right) \\
u_{ \pm}^{2}=\frac{1}{2}\left(1 \pm \sqrt{\frac{X_{+}^{2}}{X_{+}^{2}+1}}\right) . \\
\text { Now } \operatorname{the~density~of~states~}(\mathrm{DOS}) \text { is defined as: } \\
\text { DOS }(E)=\frac{1}{\pi} \operatorname{Im} G . \\
\quad \operatorname{DOS}(E)=\frac{2}{\pi^{3}\left(E+a_{3}\right)}\left[\left(X_{-}^{2}+1\right)\left(X_{+}^{2}+1\right)\right]^{\frac{-1}{4}} \\
\quad\left[K\left(v_{+}\right) K\left(u_{+}\right)-K\left(v_{-}\right) K\left(u_{-}\right)\right] \\
\left.-\left(2 a_{1}-a_{3}\right)<E<0\right),
\end{gathered}
$$

where $K\left(v_{+}\right)$and $K\left(u_{+}\right)$are the complete elliptic integrals of the first kind.

Consider the case of a tight-binding Hamiltonian $(\mathrm{TBH})$ whose perfect periodicity is destroyed due to the presence of the point defect at the $L$ site. This situation can be thought of physically as arising by substituting the host atom at the $L$-site by a foreign atom $[1,47]$ having a level lying $\varepsilon$ higher than the common level of the host atoms $(L)$. Normally, this atom is close to the host in the same series of the periodic table.

Thus, our diagonal GF of anisotropic fcc lattice for the single impurity case can be written as:

for the case where $E>2 a_{1}+a_{3}$ (outside the band)

$$
G^{0}(E)=\frac{4 K\left(k_{+}\right) K\left(k_{-}\right)}{\pi^{2}\left(E+a_{3}\right)-4 \varepsilon K\left(k_{+}\right) K\left(k_{-}\right)},
$$

while for the case of $-\left(2 a_{1}-a_{3}\right)<E<0$ (inside the band)

$$
\begin{aligned}
& G^{0}(E)=\left\{\frac{\pi^{2}}{2}\left(E+a_{3}\right)\left[\left(X_{+}^{2}+1\right)\left(X_{-}^{2}+1\right)\right]^{\frac{1}{4}}\right. \\
& \quad\left[K\left(v_{+}\right) K\left(u_{-}\right)+K\left(v_{-}\right) K\left(u_{+}\right)+i\left(K\left(v_{+}\right) K\left(u_{+}\right)\right.\right. \\
& \left.\left.-K\left(v_{-}\right) K\left(u_{-}\right)\right)\right]-\varepsilon\left[K^{2}\left(v_{+}\right)+K^{2}\left(v_{-}\right)\right]\left[K^{2}\left(u_{+}\right)\right. \\
& \left.\left.+K^{2}\left(u_{-}\right)\right]\right\} /\left\{\left[\frac{\pi^{2}}{2}\left(E+a_{3}\right)\left(\left(X_{+}^{2}+1\right)\left(X_{-}^{2}+1\right)\right)^{\frac{1}{4}}\right.\right. \\
& \left.-\varepsilon\left(K\left(v_{+}\right) K\left(u_{-}\right)+K\left(v_{-}\right) K\left(u_{+}\right)\right)\right]^{2} \\
& \left.+\varepsilon^{\prime 2}\left[K\left(v_{+}\right) K\left(u_{+}\right)-K\left(v_{-}\right) K\left(u_{-}\right)\right]^{2}\right\} .
\end{aligned}
$$

The corresponding DOS can be written as

$$
\operatorname{DOS}(E)=\left[\frac{\pi}{2}\left(E+a_{3}\right)\left[\left(X_{+}^{2}+1\right)\left(X_{-}^{2}+1\right)\right]^{\frac{1}{4}}\right.
$$




$$
\begin{aligned}
& \left.\times\left(K\left(v_{+}\right) K\left(u_{+}\right)-K\left(v_{-}\right) K\left(u_{-}\right)\right)\right] /\left\{\left[\frac{\pi^{2}}{2}\left(E+a_{3}\right)\right.\right. \\
& \left(\left(X_{+}^{2}+1\right)\left(X_{-}^{2}+1\right)\right)^{\frac{1}{4}}-\varepsilon\left(K\left(v_{+}\right) K\left(u_{-}\right)\right. \\
& \left.\left.+K\left(v_{-}\right) K\left(u_{+}\right)\right)\right]^{2}+\varepsilon^{2}\left[K\left(v_{+}\right) K\left(u_{+}\right)\right. \\
& \left.\left.-K\left(v_{-}\right) K\left(u_{-}\right)\right]^{2}\right\} \text { with }-\left(2 a_{1}-a_{3}\right)<E<0 .(2.10)
\end{aligned}
$$

The $S$-wave phase shift (i.e, $\delta_{0}$ ) is defined as the shift in the phase of the wave function due to the presence of the impurity potential, and it is defined in terms of LGF as [46]:

$$
\tan \delta_{0}=\frac{\operatorname{Im} G^{0}(E)}{\frac{1}{\varepsilon^{\prime}}-\operatorname{Re} G^{0}(E)} .
$$

Here, $\operatorname{Re} G^{0}(E)$ and $\operatorname{Im} G^{0}(E)$ refer to the real and imaginary parts of the GF inside the band respectively. After some mathematical manipulations, we obtain

$$
\begin{gathered}
\tan \delta_{0}=K\left(v_{+}\right) K\left(u_{+}\right)-K\left(v_{-}\right) K\left(u_{-}\right) \\
\left(\frac{\pi^{2}\left(E+a_{3}\right)\left[\left(X_{+}^{2}+1\right)\left(X_{-}^{2}+1\right)\right]^{\frac{1}{4}}}{2 \varepsilon}\right. \\
\left.-\left(K\left(v_{+}\right) K\left(u_{-}\right)+K\left(v_{-}\right) K\left(u_{+}\right)\right)\right)^{-1} .
\end{gathered}
$$

The cross-section (i.e., $\sigma$ ) is defined as [46]:

$$
\sigma=\frac{4 \pi}{P^{2}} \frac{\left[\operatorname{Im} G^{0}(E)\right]^{2}}{-\left[\operatorname{Re} G^{0}(E)-\frac{1}{\varepsilon}\right]^{2}+\left[\operatorname{Im} G^{0}(E)\right]^{2}} .
$$

Here, $P$ refers to the electron momentum.

Therefore, the cross-section becomes

$$
\begin{aligned}
\sigma & =\frac{4 \pi}{P^{2}}\left[K\left(v_{+}\right) K\left(u_{+}\right)-K\left(v_{-}\right) K\left(u_{-}\right)\right]^{2} \\
& /\left\{\left[K\left(v_{+}\right) K\left(u_{-}\right)+K\left(v_{-}\right) K\left(u_{+}\right)\right.\right. \\
& \left.-\frac{\pi^{2}\left(E+a_{3}\right)\left[\left(X_{+}^{2}+1\right)\left(X_{-}^{2}+1\right)\right]^{\frac{1}{4}}}{2 \varepsilon}\right]^{2} \\
& \left.+\left[K\left(v_{+}\right) K\left(u_{+}\right)-K\left(v_{-}\right) K\left(u_{-}\right)\right]^{2}\right\} .
\end{aligned}
$$

\subsection{Special cases}

The following special cases are given:

1. When $a_{1}=a_{2}=a_{3}=1$, we recover the fcc lattice.

2. When $a_{1}=1$ and $a_{3}=0$

The diagonal GF outside the band has the form

$$
G^{0}(E)=\frac{4}{\pi^{2} E} K^{2}(k),
$$

with $|E|>2$, and

$$
k^{2}=\frac{1}{2}\left(1-\sqrt{1-\frac{4}{E^{2}}}\right) .
$$

The GF outside and inside the band can be written as (see Appendix A):

$$
G^{0}(E)=\left\{\begin{array}{l}
\frac{4}{\pi^{2} E} K^{2}(k), \quad|E|>2, \\
\frac{1}{\pi^{2}}\left(2 K\left(u_{+}\right) K\left(u_{-}\right)\right. \\
\left.\quad+\mathrm{i}\left[K^{2}\left(u_{+}\right)-K^{2}\left(u_{-}\right)\right]\right), \quad-2<E<0,
\end{array}\right.
$$

$$
\begin{aligned}
& X_{+}=X_{-}=\left(-\sqrt{\frac{4-E^{2}}{E^{2}}}\right), \\
& u_{ \pm}^{2}=v_{ \pm}^{2}=\frac{1}{2}\left(1 \pm \sqrt{1-\frac{E^{2}}{4}}\right) .
\end{aligned}
$$

Therefore, the DOS is

$$
\operatorname{DOS}=\frac{1}{\pi^{3}}\left[K^{2}\left(u_{+}\right)-K^{2}\left(u_{-}\right)\right], \quad-2<E<0,
$$

where $K\left(v_{ \pm}\right)$and $K\left(u_{ \pm}\right)$are the complete elliptic integrals of the first kind.

Thus, our diagonal GF of anisotropic face centered cubic lattice for the single impurity case can be written as

$$
G^{0}(E)= \begin{cases}\frac{4 K^{2}(k)}{\frac{\pi^{2} E}{4}-\varepsilon^{\prime} K^{2}(k)}, & E>2, \\ \left\{\pi ^ { 2 } \left[2 K\left(u_{+}\right) K\left(u_{-}\right)\right.\right. & \\ & \left.+\mathrm{i}\left(K^{2}\left(u_{+}\right)-K^{2}\left(u_{-}\right)\right)\right] \\ & \left.-\varepsilon^{\prime}\left[K^{2}\left(u_{+}\right)+K^{2}\left(u_{-}\right)\right]^{2}\right\} \\ & \\ /\left[\pi^{2}-2 \varepsilon^{\prime} K\left(u_{+}\right) K\left(u_{-}\right)\right]^{2} & \\ +\varepsilon^{\prime 2}\left[K^{2}\left(u_{+}\right)-K^{2}\left(u_{-}\right)\right]^{2}, & -2<E<0 .\end{cases}
$$

The corresponding DOS can be written as

$\operatorname{DOS}(E)=$

$$
\begin{aligned}
& \frac{\pi\left(K^{2}\left(u_{+}\right)-K^{2}\left(u_{-}\right)\right)}{\left[\pi^{2}-2 \varepsilon K\left(u_{+}\right) K\left(u_{-}\right)\right]^{2}+\varepsilon^{2}\left[K^{2}\left(u_{+}\right)-K^{2}\left(u_{-}\right)\right]^{2}}, \\
& -2<E<0 .
\end{aligned}
$$

The $S$-wave phase shift, $\delta_{0}$, is

$$
\tan \delta_{0}=\frac{K^{2}\left(u_{+}\right)-K^{2}\left(u_{-}\right)}{\frac{\pi^{2}}{\varepsilon}-2 K\left(u_{+}\right) K\left(u_{-}\right)} .
$$

The cross-section, $\sigma$, is

$$
\begin{aligned}
& \sigma= \\
& \frac{4 \pi}{P^{2}} \frac{\left[K^{2}\left(u_{+}\right)-K^{2}\left(u_{-}\right)\right]^{2}}{-\left[2 K\left(u_{+}\right) K\left(u_{-}\right)-\frac{\pi^{2}}{\varepsilon}\right]^{2}+\left[K^{2}\left(u_{+}\right)-K^{2}\left(u_{-}\right)\right]^{2}} .
\end{aligned}
$$

\section{Results and discussion}

Our results for the anisotropic fcc lattice are shown in Figs. 1-9. Figures 1, 2 show real and imaginary parts of GF for the pure lattice. The figures show logarithmic behavior. Figure 3 shows the DOS for the pure lattice. The DOS has the same behavior as above apart from a constant. The figure shows that the function is symmetric (even function).

Figure 4 shows the DOS for the anisotropic fcc lattice with single impurity for different potential strengths $\varepsilon(-0.6,-0.3,0.0,0.3$, and 0.6$)$. For $\varepsilon=0.0$ it falls off exponentially. The peak value varies with the potential strengths and reaches its maximum at $\varepsilon=0.3$, also the divergence of the density of states removed by adding such impurities. Figure 5 shows the DOS in threedimensions with one axis representing potential strengths $\varepsilon$ varying between -1 and 1 (arbitrary units) whereas the second axis is energy scale varying between -1 and 1 as indicated in the formalism. 


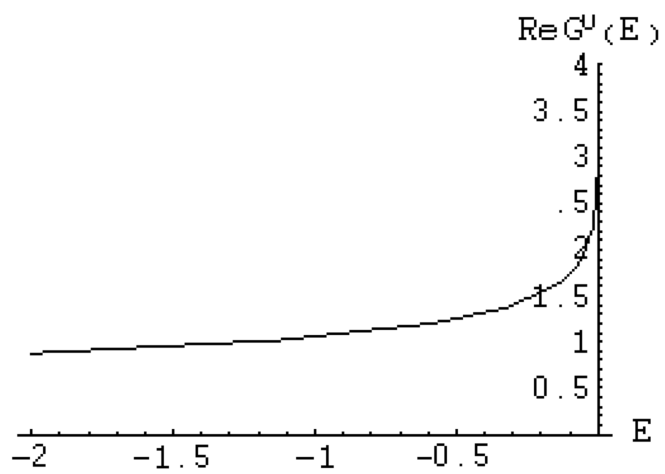

Fig. 1. Real part GF for the perfect anisotropic fcc lattice.

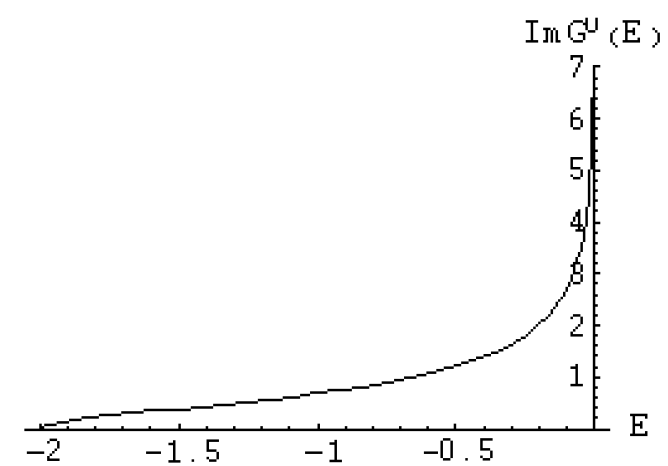

Fig. 2. Imaginary part GF for the perfect anisotropic fcc lattice.

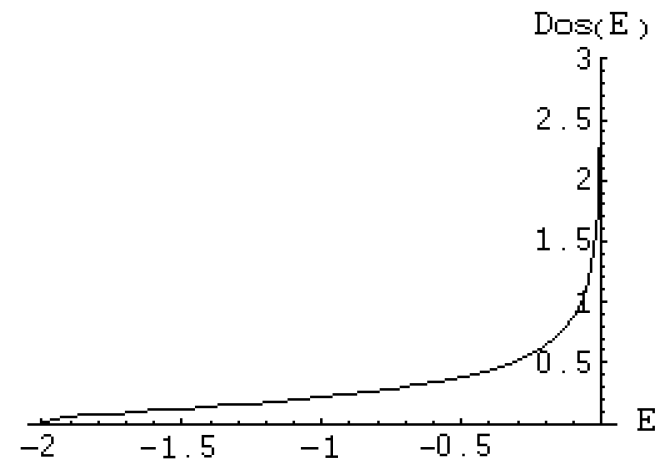

Fig. 3. The DOS for the perfect anisotropic fcc lattice.

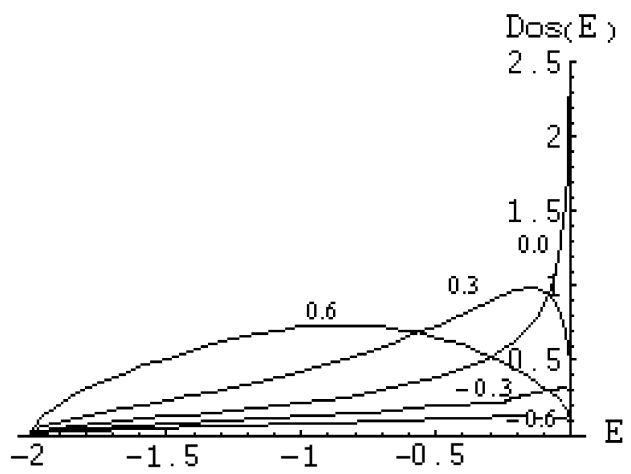

Fig. 4. The DOS for the anisotropic fcc lattice with single impurity for different potential strengths $\varepsilon(-0.6$, $-0.3,0.0,0.3$, and 0.6 ).

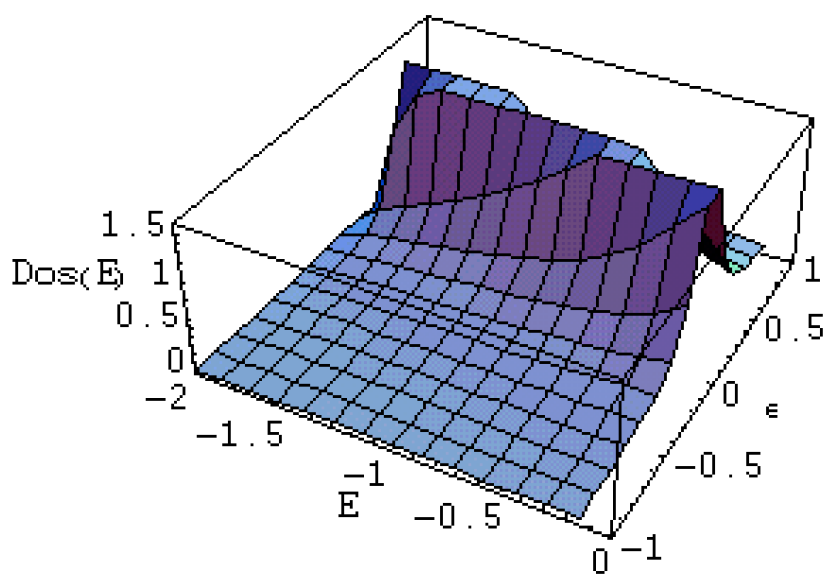

Fig. 5. Three-dimensional DOS for the anisotropic fcc lattice with single impurity for different potential strengths $\varepsilon$ varying between -1 and 1 (arbitrary units).

The phase shift (i.e, $\left.\delta_{0}\right)$ is defined as the shift in the phase of the wave function due to the presence of the impurity potential.

Figure 6 displays $\delta_{0}$ for the anisotropic fcc lattice with single impurity for different potential strengths $\varepsilon(-0.6$, $0.3,0.0,0.3$, and 0.6$)$. For $\varepsilon=0.0, \delta_{0}$ vanishes as potential is turned off (perfect lattice). The phase shift is always negative for all negative potential strengths $\varepsilon$. In the range between $\varepsilon=0.00$ and $\varepsilon=0.3, \delta_{0}$ is positive. In the range where $\varepsilon$ varies between 0.3 and 1.0 we have discontinuity as shown in Fig. $6, \delta_{0}$ displays into two regions around the discontinuity point, right hand region is negative and it increases if $\varepsilon$ increases, the left hand region is positive and it decreases if $\varepsilon$ increases (discontinuity point moves to the left).

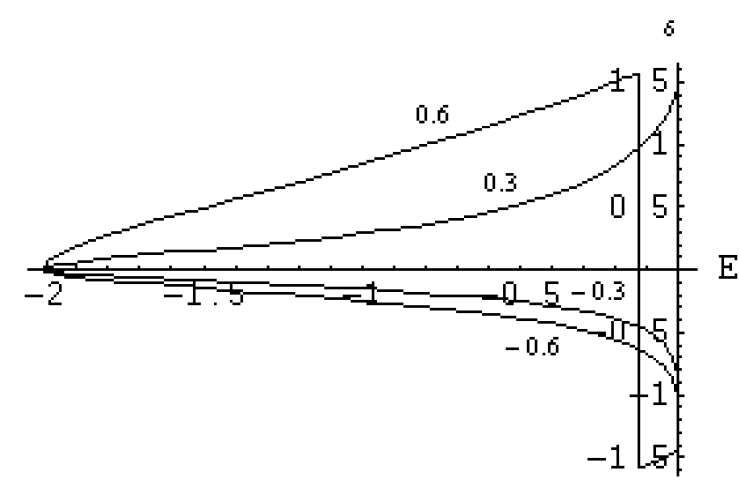

Fig. 6. The phase shift, $\delta_{0}$, for the anisotropic fcc lattice with single impurity for different potential strengths $\varepsilon(-0.6,-0.3,0.0,0.3$, and 0.6$)$.

Figure 7 shows the phase shift in three dimensions for the anisotropic fcc lattice with single impurity for different potential strengths $\varepsilon$ varying between -1 and 1 (arbitrary units).

The cross-section (i.e., $\sigma$ ) is defined as the area an impurity atom presents to the incident electron. 


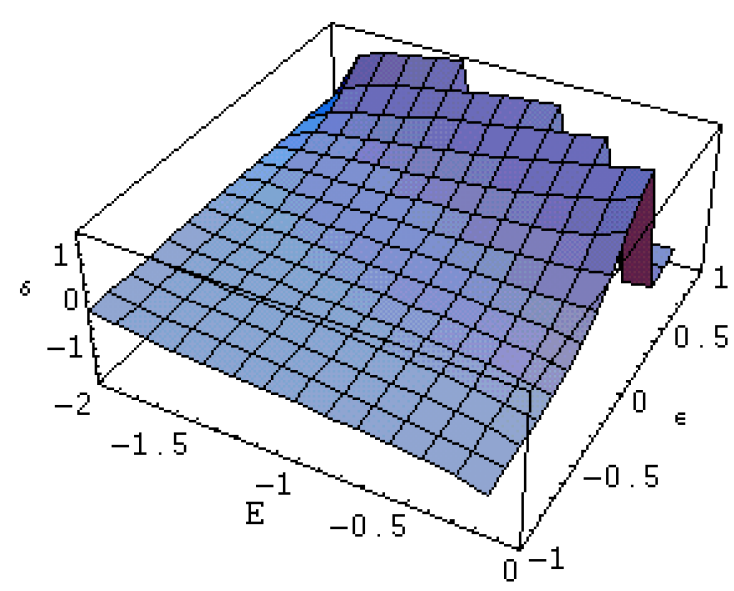

Fig. 7. The phase shift, $\delta_{0}$, in three dimensions for the anisotropic fcc lattice with single impurity for different potential strengths $\varepsilon$ varying between -1 and 1 (arbitrary units).

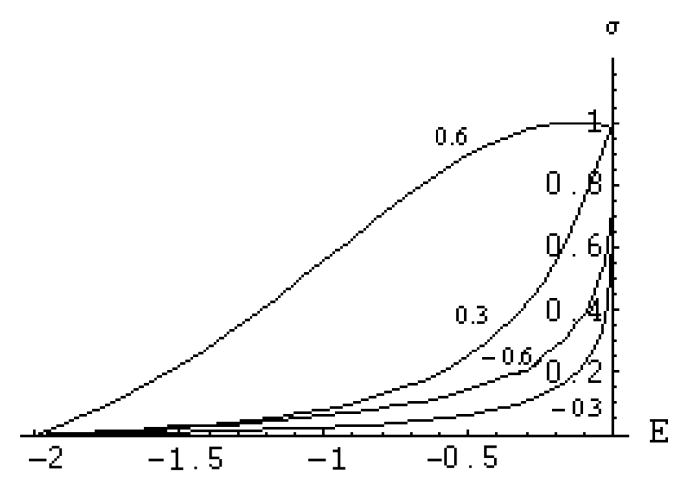

Fig. 8. The cross section, $\sigma$, for the anisotropic fcc lattice with single impurity for different potential strengths $\varepsilon(-0.6,-0.3,0.0,0.3$, and 0.6$)$.

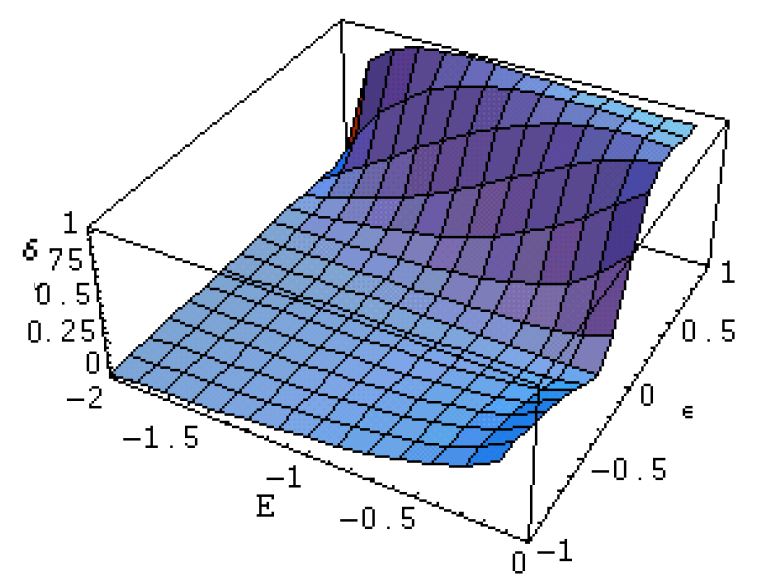

Fig. 9. The cross section, $\sigma$, in three dimensions for the anisotropic fcc lattice with single impurity for different potential strengths $\varepsilon$ varying between -1 and 1 (arbitrary units).
Figure 8 shows the cross-section for single substitutional impurity with different potential strength $\varepsilon$, the peak value varies with the potential strength and reaches its maximum for all values $\varepsilon>0.3$, in the range where $\varepsilon$ varies between 0.0 and 0.3 the peak value increases if $\varepsilon$ increases, in the range between $\varepsilon=0.0$ and -1.0 the peak value increases if $\varepsilon$ decreases. The values are all positive since $\sigma$ can be viewed as a sort of probability. It is related to some physical quantities such as the conductivity in metals.

Figure 9 shows the cross-section in three dimensions for the anisotropic fcc lattice with single impurity for different potential strengths $\varepsilon$ varying between -1 and 1 (arbitrary units).

\section{Appendix A}

\section{Derivation of GF for the fcc lattice inside the band}

In this Appendix we derive an expression for the GF inside the band in order to calculate the DOS which is related to some important physical quantities in terms of the complete elliptic integral of the first kind. The GF for the anisotropic fcc lattice outside the band is given by $[10-16]$ :

$$
G^{0}(E)=\frac{4}{\pi^{2}\left(E+a_{3}\right)} K\left(k_{+}\right) K\left(k_{-}\right), E>2 a_{1}+a_{3},
$$

where

$$
\begin{gathered}
k_{ \pm}^{2}=\frac{1}{2}\left(1 \mp 4 \frac{\sqrt{\frac{a_{3}}{a_{1}^{2}} E\left(1+\frac{a_{3}}{a_{1}^{2}} E\right)}}{\left(E+a_{3}\right)^{2}}\right. \\
\left.-\frac{\sqrt{\left(E+a_{3}\right)^{2}-4 \frac{a_{3}}{a_{1}^{2}} E} \sqrt{\left(E+a_{3}\right)^{2}-4\left(1+\frac{a_{3}}{a_{1}^{2}} E\right)}}{\left(E+a_{3}\right)^{2}}\right)
\end{gathered}
$$

or in the range $E$ enclosed between $-\left(2 a_{1}-a_{3}\right)$ and 0

$$
k_{ \pm}^{2}=\frac{1}{2}\left(1+Z_{\mp}\right), \quad-\left(2 a_{1}-a_{3}\right)<E<0,
$$

where

$$
\begin{gathered}
Z_{\mp}=\mathrm{i} X_{\mp} \\
X_{\mp}=\frac{\sqrt{-E}}{\left(E+a_{3}\right)^{2}} \\
\times\left(-\sqrt{\frac{\left[\left(E+a_{3}\right)^{2}-4 \frac{a_{3}}{a_{1}^{2}} E\right]\left[4\left(1+\frac{a_{3}}{a_{1}^{2}} E\right)-\left(E+a_{3}\right)^{2}\right]}{-E}}\right. \\
\mp 4 \sqrt{\left.\frac{a_{3}}{a_{1}^{2}}\left(1+\frac{a_{3}}{a_{1}^{2}} E\right)\right)} \cdot
\end{gathered}
$$

The complete elliptic integral of the first kind is expressed as 


$$
K(k)=\frac{\pi}{2}{ }_{2} F_{1}\left(\frac{1}{2}, \frac{1}{2}, 1, k^{2}\right)
$$

where ${ }_{2} F_{1}\left(\frac{1}{2}, \frac{1}{2}, 1, k^{2}\right)$ is the Gauss hypergeometric function.

Substituting (A.6) in (A.1) we have

$$
G^{0}(E)=\frac{{ }_{2} F_{1}\left(\frac{1}{2}, \frac{1}{2} ; 1 ; k_{+}^{2}\right){ }_{2} F_{1}\left(\frac{1}{2}, \frac{1}{2} ; 1 ; k_{-}^{2}\right)}{E+a_{3}}
$$

Using the following transformations [19]:

$$
\begin{gathered}
{ }_{2} F_{1}\left(\frac{1}{2}, \frac{1}{2} ; 1 ; \frac{1+Z_{\mp}}{2}\right)=\frac{\Gamma\left(\frac{1}{2}\right)}{\left(\Gamma\left(\frac{3}{4}\right)\right)^{2}}{ }_{2} F_{1}\left(\frac{1}{4}, \frac{1}{4} ; \frac{1}{2} ; Z_{\mp}^{2}\right) \\
+2 Z_{\mp} \frac{\Gamma\left(\frac{1}{2}\right)}{\left(\Gamma\left(\frac{1}{4}\right)\right)^{2}}{ }_{2} F_{1}\left(\frac{3}{4}, \frac{3}{4} ; \frac{3}{2} ; Z_{\mp}^{2}\right)
\end{gathered}
$$

with

$$
\begin{aligned}
& { }_{2} F_{1}\left(a, b ; c ; Z_{\mp}^{2}\right)= \\
& \left(1-Z_{\mp}^{2}\right)^{-a}{ }_{2} F_{1}\left(a, c-b ; c ; \frac{Z_{\mp}^{2}}{Z_{\mp}^{2}-1}\right) \\
& \frac{2 \Gamma\left(\frac{1}{2}\right)}{\left(\Gamma\left(\frac{3}{4}\right)\right)^{2}}{ }_{2} F_{1}\left(\frac{1}{4}, \frac{1}{4} ; \frac{1}{2} ; \frac{Z_{\mp}^{2}}{Z_{\mp}^{2}-1}\right)= \\
& { }_{2} F_{1}\left(\frac{1}{2}, \frac{1}{2} ; 1 ; \frac{1}{2}\left(1+\sqrt{\frac{Z_{\mp}^{2}}{Z_{\mp}^{2}-1}}\right)\right) \\
& +{ }_{2} F_{1}\left(\frac{1}{2}, \frac{1}{2} ; 1 ; \frac{1}{2}\left(1-\sqrt{\frac{Z_{\mp}^{2}}{Z_{\mp}^{2}-1}}\right)\right), \\
& \frac{2 \Gamma\left(-\frac{1}{2}\right)}{\left[\Gamma\left(\frac{1}{4}\right)\right]^{2}} \sqrt{\frac{Z_{\mp}^{2}}{Z_{\mp}^{2}-1}}{ }_{2} F_{1}\left(\frac{3}{4}, \frac{3}{4} ; \frac{3}{2} ; \frac{Z_{\mp}^{2}}{Z_{\mp}^{2}-1}\right)= \\
& { }_{2} F_{1}\left(\frac{1}{2}, \frac{1}{2} ; 1 ; \frac{1}{2}\left(1-\sqrt{\frac{Z_{\mp}^{2}}{Z_{\mp}^{2}-1}}\right)\right) \\
& -{ }_{2} F_{1}\left(\frac{1}{2}, \frac{1}{2} ; 1 ; \frac{1}{2}\left(1+\sqrt{\frac{Z_{\mp}^{2}}{Z_{\mp}^{2}-1}}\right)\right) .
\end{aligned}
$$

Substituting (A.8), (A.9),(A.10), and (A.11) in (A.7), then we obtain

$$
\begin{aligned}
& G^{0}(E)=\frac{1}{2\left(E+a_{3}\right)}\left[\left(X_{+}^{2}+1\right)\left(X_{-}^{2}+1\right)\right]^{\frac{-1}{4}} \\
& \quad \times\left(K\left(v_{+}\right) K\left(u_{-}\right)+K\left(v_{-}\right) K\left(u_{+}\right)+\mathrm{i}\left(K\left(v_{+}\right) K\left(u_{+}\right)\right.\right. \\
& \left.\left.\quad-K\left(v_{-}\right) K\left(u_{-}\right)\right)\right),
\end{aligned}
$$

where

$$
\begin{aligned}
& v_{ \pm}^{2}=\frac{1}{2}\left(1 \pm \sqrt{\frac{X_{-}^{2}}{X_{-}^{2}+1}}\right) . \\
& u_{ \pm}^{2}=\frac{1}{2}\left(1 \pm \sqrt{\frac{X_{+}^{2}}{X_{+}^{2}+1}}\right) .
\end{aligned}
$$

If we have a single impurity then GF is defined as [1]:

$$
G(E)=\frac{G^{0}(L, L, E)}{1-\varepsilon G^{0}(L, L, E)} .
$$

After some mathematical manipulation Eq. (A.15) becomes

$$
\begin{aligned}
& G(E)=\left\{\frac{\pi^{2}}{2}\left(E+a_{3}\right)\left[\left(X_{+}^{2}+1\right)\left(X_{-}^{2}+1\right)\right]^{\frac{1}{4}}\right. \\
& \times\left[K\left(v_{+}\right) K\left(u_{-}\right)+K\left(v_{-}\right) K\left(u_{+}\right)+\mathrm{i}\left(K\left(v_{+}\right) K\left(u_{+}\right)\right.\right. \\
& \left.\left.-K\left(v_{-}\right) K\left(u_{-}\right)\right)\right]-\varepsilon\left[K^{2}\left(v_{+}\right)+K^{2}\left(v_{-}\right)\right]\left[K^{2}\left(u_{+}\right)\right. \\
& \left.\left.+K^{2}\left(u_{-}\right)\right]\right\} /\left\{\left[\frac{\pi^{2}}{2}\left(E+a_{3}\right)\left[\left(X_{+}^{2}+1\right)\left(X_{-}^{2}+1\right)\right]^{\frac{1}{4}}\right.\right. \\
& \left.-\varepsilon^{\prime}\left(K\left(v_{+}\right) K\left(u_{-}\right)+K\left(v_{-}\right) K\left(u_{+}\right)\right)\right]^{2} \\
& \left.\left.+\varepsilon^{2}\left[K\left(v_{+}\right) K\left(u_{+}\right)-K\left(v_{-}\right) K\left(u_{-}\right)\right)\right]^{2}\right\} .
\end{aligned}
$$

Thus, the $S$-phase shift, and scattering cross-section can be evaluated in terms of complete elliptic integrals of the first kind as shown in the text.

\section{References}

[1] E.N. Economou, Green Function in Quantum Physics, 2nd ed., Springer-Verlag, Berlin 1983.

[2] S. Katsura, T. Morita, S. Inawashiro, T. Horiguci, Y. Abe, J. Math. Phys. 12, 892 (1971).

[3] T. Morita, T. Horiguchi, J. Phys. A Math. Gen. 5 , 67 (1972).

[4] A.L. Fetter, J.D. Walecka, Quantum Theory of Many-Particle Systems, McGraw-Hill, New York 1971.

[5] B.M. McCoy, T.T. Wu, Phys. Rev. D 18, 1253 (1978).

[6] N.W. Dalton, D.W. Wood, Proc. Phys. Soc. (London) 90, 4591 (1967).

[7] M. Lax, Phys. Rev. 85, 621 (1952).

[8] E. Montroll, G. Weiss, J. Math. Phys. 6, 167 (1965).

[9] B.D. Hughes, Physica 134A, 443 (1986).

[10] G.L. Montet, Phys. Rev. B 7, 650 (1973).

[11] G.F. Koster, D.C. Slater, Phys. Rev. 96, 1208 (1954).

[12] J. Cserti, Am. J. Phys. 68, 896 (2000).

[13] J. Cserti, G. David, A. Piroth, Am. J. Phys. 70, 153 (2002).

[14] J.H. Asad, R.S. Hijjawi, A.J. Sakaji, J.M. Khalifeh, Int. J. Theor. Phys. 43, 2223 (2004).

[15] J.H. Asad, R.S. Hijjawi, A.J. Sakaji, J.M. Khalifeh, Int. J. Theor. Phys. 44, 471 (2005).

[16] J.H. Asad, A.J. Sakaji, R.S. Hijjawi, J.M. Khalifeh, Int. J. Mod. Phys. B. 19, 3713 (2005).

[17] J.H. Asad, A.J. Sakaji, R.S. Hijjawi, J.M. Khalifeh, Eur. Phys. J. B 52, 365 (2006).

[18] R.S. Hijjawi, J.H. Asad, A.J. Sakaji, J.M. Khalifeh, Int. J. Mod. Phys. B 21, 199 (2007).

[19] J.H. Asad, Mod. Phys. Lett. B 21, 139 (2007).

[20] J.H. Asad, R.S. Hijjawi, A.J. Sakaji, J.M. Khalifeh, Europ. Phys. J. Appl. Phys. 40, 257 (2007).

[21] R.S. Hijjawi, J.A. Asad, A.J. Sakaji, M. Al-Sabayleh, J.M. Khalifeh, Europ. Phys. J. Appl. Phys. 41, 111 (2008). 
[22] J.H. Asad, R.S. Hijjawi, A.J. Sakaji, J.M. Khalifeh, Mod. Phys. Lett. B 24, 695 (2010).

[23] J.H. Asad, A.A. Diab, R.S. Hijjawi, J.M. Khalifeh, Europ. Phys. J. Plus 128, 1 (2013).

[24] J.H. Asad, J. Statist. Phys. 150, 1177 (2013).

[25] J.H. Asad, Mod. Phys. Lett. B 27, 1350112 (2013).

[26] J.H. Asad, A.A. Diab, M.Q. Owaidat, J.M. Khalifeh, Acta Phys. Pol. A 126, 777 (2014).

[27] S. Katsura, S. Inawashiro, J. Math. Phys. 12, 1622 (1971).

[28] T. Morita, J. Math. Phys. 12, 1744 (1971).

[29] G.S. Joyce, J. Phys. A Math. Gen. 5, L65 (1972).

[30] T. Horiguchi, J. Phys. Soc. Jpn. 30, 1261 (1971).

[31] S. Katsura, S. Inawashiro, J. Math. Phys. 12, 1622 (1971).

[32] S. Katsura, T. Horiguchi, J. Math. Phys. 12, 230 (1971).

[33] M.L. Glasser, J. Boersma, J. Phys. A Math. Gen. 33, 5017 (2000).

[34] G. Iwata, Nat. Soc. Rep. (Ochanomizu Univ.) 20, 13 (1969).

[35] K. Mano, J. Math. Phys. 16, 1726 (1975).
[36] A. Sakaji, R.S. Hijjawi, N. Shawagfeh, J.M. Khalifeh, J. Math. Phys. 43, 235 (2002).

[37] A. Sakaji, R.S. Hijjawi, N. Shawagfeh, J.M. Khalifeh, J. Theor. Phys. 41, 973 (2002).

[38] R.S. Hijjawi, J.M. Khalifeh, J. Theor. Phys. 41, 1769 (2002).

[39] R.S. Hijjawi, J.H. Asad, A. Sakaji, J.M. Khalifeh, J. Theor. Phys. 43, (2004).

[40] S.L. Kalla, S. Conde, J.H. Hubbell, Appl. Anal. 22, 273 (1986).

[41] G.S. Joyce, J. Phys. C Solid State Phys. 4, L53 (1971).

[42] T. Morita, T. Horiguci, J. Math. Phys. 12, 986 (1971).

[43] T. Morita, T. Horiguci, J. Math. Phys. 12, 981 (1971).

[44] M. Inoue, J. Math. Phys. 15, 704 (1974).

[45] K. Mano, J. Math. Phys. 16, 1726 (1975).

[46] K. Mano, J. Math. Phys. 15, 2175 (1974).

[47] S. Doniach, E.H. Sondheimer, Green's Functions for Solid State Physicists, Benjamin, Reading ( MA) 1974. 